\title{
Impactos da pandemia do COVID-19 na prática de atividades físicas: Percepção de praticantes de Spinning na participação em treinos remotos
}

\author{
Impacts of the COVID-19 pandemic on the practice of physical activities: Perception of Spinning \\ practitioners in participation in remote training
}

Impactos de la pandemia COVID-19 en la práctica de actividades físicas: Percepción de los practicantes de spinning en la participación en el entrenamiento remoto

Recebido: 12/07/2021 | Revisado: 17/07/2021 | Aceito: 22/07/2021 | Publicado: 29/07/2021

\author{
Thiago Santos de Araújo \\ ORCID: https://orcid.org/0000-0001-9877-6218 \\ Universidade Federal do Rio Grande do Norte, Brasil \\ E-mail: thiagosantos82@hotmail.com \\ Ana Carolina Patrício de Albuquerque Sousa \\ ORCID: https://orcid.org/0000-0001-7517-8792 \\ Universidade Federal do Rio Grande do Norte, Brasil \\ E-mail: acapas@gmail.com \\ Dejeane Domingos Soares \\ ORCID: https://orcid.org/0000-0001-9826-8168 \\ Universidade Potiguar, Brasil \\ E-mail: dejeaneds@gmail.com \\ Tatiane Isabela de Araújo Leite \\ ORCID: https://orcid.org/0000-0002-4820-485X \\ Universidade do Estado do Rio Grande do Norte, Brasil \\ E-mail: tatianeisabela22@hotmail.com \\ Lúcio Flávio Pereira \\ ORCID: https://orcid.org/0000-0001-5158-6302 \\ Universidade Potiguar, Brasil \\ E-mail: ultatrompete@hotmail.com
}

\begin{abstract}
Resumo
Segundo a World Health Organization uma nova condição adversa de saúde afeta e se espalha de forma pandêmica em diferentes continentes com transmissão sustentada de pessoa para pessoa, tratando-se do SARS-CoV-2. O objetivo deste estudo é analisar a percepção de praticantes de Spinning na participação em aulas remotas durante o período de isolamento da pandemia do COVID-19. Este estudo trata-se de um relato de experiência como instrutor de aula remotas de Spinning, realizado no primeiro semestre de 2020, onde foi aplicado um formulário da plataforma Google Forms com a participação de 30 indivíduos, com questionamentos sobre a experiência de realizar treinos remotos de Spinning. Dentre os resultados encontrados observou-se que todos os participantes eram do sexo feminino, que $68,2 \%$ dos usuários relataram que a experiência de se exercitarem em casa foi excelente; a maioria dos usuários participavam das aulas 2 ou 3 vezes na semana; quanto ao uso da plataforma digital durante as aulas virtuais, a maioria julgou como ótima a ferramenta. Diante do pressuposto pode-se concluir que a experiência de participar de aulas remotas de Spinninig em casa, no momento de isolamento social imposta pelo COVID-19, foi muito benéfica para saúde física e emocional dos praticantes, e que ações como essas devem ser encorajadas e estimuladas, visto a importância de nos mantermos ativos e cuidando da saúde mesmo em períodos de pandemia e isolamento social.
\end{abstract}

Palavras-chave: Spinning; Isolamento social; COVID-19; Treino remoto.

\begin{abstract}
According to the World Health Organization, a new adverse health condition affects and spreads pandemicly in different continents with sustained transmission from person to person, namely SARS-CoV-2. The aim of this study is to analyze the perception of Spinning practitioners in participating in remote classes during the COVID-19 pandemic isolation period. This study is an experience report as a remote Spinning class instructor, carried out in the first half of 2020, where a form from the Google Forms platform was applied with the participation of 30 individuals, with questions about the experience of performing remote training Spinning. Among the results found, it was observed that all participants were female, that $68.2 \%$ of users reported that the experience of exercising at home was excellent; most users attended classes 2 or 3 times a week; regarding the use of the digital platform during virtual classes, most judged the tool to be excellent. Given the assumption, it can be concluded that the experience of participating in remote Spinninig classes at home, at the time of social isolation imposed by COVID-19, was very beneficial to the
\end{abstract}


physical and emotional health of practitioners, and that actions such as these should be encouraged and stimulated, given the importance of staying active and taking care of our health even in periods of pandemic and social isolation.

Keywords: Spinning; Social isolation; COVID-19; Remote training.

\section{Resumen}

Según la Organización Mundial de la Salud, una nueva condición de salud adversa afecta y se propaga de manera pandémica en diferentes continentes con transmisión sostenida de persona a persona, a saber, el SARS-CoV-2. El objetivo de este estudio es analizar la percepción de los practicantes de Spinning al participar en clases remotas durante el período de aislamiento pandémico de COVID-19. Este estudio es un relato de experiencia como instructor de clase de Spinning remoto, realizado en el primer semestre de 2020, donde se aplicó un formulario de la plataforma Google Forms con la participación de 30 personas, con preguntas sobre la experiencia de realizar entrenamiento remoto de Spinning. Entre los resultados encontrados, se observó que todos los participantes eran mujeres, que el $68,2 \%$ de los usuarios informó que la experiencia de hacer ejercicio en casa fue excelente; la mayoría de usuarios asistían a clases 2 o 3 veces por semana; con respecto al uso de la plataforma digital durante las clases virtuales, la mayoría consideró que la herramienta era excelente. Dado el supuesto, se puede concluir que la experiencia de participar en clases remotas de Spinninig en casa, en el momento del aislamiento social impuesto por COVID-19, fue muy beneficiosa para la salud física y emocional de los practicantes, y que acciones como estas Se debe alentar y estimular, dada la importancia de mantenernos activos y cuidar nuestra salud incluso en períodos de pandemia y aislamiento social.

Palabras clave: Spinning; Aislamiento social; COVID-19; Entrenamiento remoto.

\section{Introdução}

Segundo a World Health Organization (WHO, 2020) uma pandemia é a disseminação mundial de uma nova doença. O termo é utilizado quando uma determinada condição adversa de saúde afeta de forma epidêmica uma região e se espalha por diferentes continentes com transmissão sustentada de pessoa para pessoa. Historicamente, o mundo já enfrentou diversas pandemias por longos períodos como a Gripe Espanhola, que durou cerca de dois anos e foi considerada até o momento a mais mortal da história da humanidade (WHO, 2020). A última pandemia que surpreendeu o mundo foi a H1N1 no ano de 2009, todos os cidadãos e principalmente os profissionais de saúde tiveram que se adaptar àquela nova realidade. No primeiro trimestre de 2020, o mundo vivenciou uma nova pandemia, conhecida como o novo Coronavírus (SARS-CoV-2), mais grave e de rápida e incontrolável disseminação.

Os Coronavírus são vírus de RNA envelopados amplamente distribuídos entre humanos, além de outros mamíferos e aves. O novo Coronavírus (SARS-CoV-2) é uma cepa da família coronavírus que ainda não havia sido detectada em humanos, um novo vírus com capacidade de contágio alta, onde cada pessoa contaminada contagia em média duas pessoas. A transmissão de pessoa a pessoa do SARS-CoV-2, ocorre por meio de gotículas respiratórias (aerossol, fala, tosse ou espirro) e ainda pelo contato direto com pessoas infectadas, ou indireto por meio das mãos, objetos ou superfícies contaminadas (Werneck \& Carvalho, 2020). Devido à facilidade de contaminação, o atual momento é atípico no Brasil e no mundo, com populações orientadas a não saírem de casa e ficarem em isolamento social. As pessoas recebem inúmeras informações, de diversas fontes, a todo tempo, surgindo à preocupação com a saúde e bem estar da população seja ela física ou mental (Brasil, 2020; Ornell, Schuch, Sordi, Kessler, 2020).

A ociosidade, o comodismo e principalmente a adequação a uma nova rotina, pode ser um desafio para várias pessoas, por essa razão adequar nessa rotina à prática de exercícios físicos auxiliará na preservação da saúde do indivíduo ${ }^{4}$. Sabe-se o quão importante é a prática regular de exercícios físicos e os reais benefícios para seus praticantes, que vão desde a melhoria da qualidade do sono, do perfil lipídico e da sensibilidade à insulina, até o fortalecimento muscular e imunológico (Rosa, Bicudo, Vaisberg, 2020; Santos \& Santos, 2010). São vários os estudos publicados evidenciando a importância de manter-se ativo e saudável, pois além de manter corpo e a mente saudáveis, o exercício físico é atuante na prevenção e tratamento de Doenças Crônicas Não-Transmissíveis (DCNT), como diabetes, hipertensão arterial, doenças respiratórias, entre outras (Terra et. al., 2012; Brasil, 2015; Pires, 2020).

O exercício físico é conhecido por ter grande impacto no funcionamento do sistema imunológico. Exercícios moderados a intensos, feitos regularmente de forma controlada, tem mostrado melhora da resposta imunológica, diminuição do estado inflamatório crônico e melhora dos diversos marcadores imunológicos de doenças como câncer, HIV, doenças 
cardiovasculares, diabetes e obesidade (Leandro et. al., 2007). A manutenção do exercício físico no contexto atual é essencial na prevenção de comorbidades visto que, a pandemia juntamente à restrição de mobilidade e isolamento social torna a ociosidade mais constante. A prática regular de exercícios físicos, que antes era comprometida pela rotina atribulada, tornou-se indispensável nesse período de isolamento social (SBMEE, 2020; Krinski, 2010). Durante sua prática, vários são os hormônios liberados, em especial a endorfina, que tem a função protetora de controlar a ansiedade e diminuir o estresse, e a serotonina, cuja atuação está relacionada ao humor, sono e apetite, ambos proporcionando a sensação de bem-estar.

Um artigo publicado no jornal britânico The Guardian (2020) afirma que o exercício, além de influenciar o cérebro de diferentes maneiras, mantém a mente saudável, melhorando a memória, concentração e humor. A união de exercícios físicos e relaxamento é uma ferramenta valiosa para manter a calma e continuar a proteger a saúde durante esse período de isolamento. A American College of Sports Medicine (ACMS, 2020) recomenda para indivíduos assintomáticos a prática de 150 minutos de atividade física de intensidade moderada ou 75 minutos de intensidade vigorosa por semana, ou uma combinação de ambos. Essas recomendações ainda podem ser alcançadas mesmo em casa, sem equipamento especial e com espaço limitado.

Diante do pressuposto o objetivo deste estudo é analisar a percepção de praticantes de Spinning em aulas remotas em casa durante o período de isolamento social imposto pela pandemia do COVID-19.

\section{Metodologia}

A bicicleta foi uma invenção revolucionária, e até os dias de hoje pedalar sempre foi uma curtição para todas as idades e sexos, e a ideia de levar uma bicicleta estacionária para dentro de uma sala, estúdio ou ginásio afim de promover uma aula ou uma sessão de treinamento elaborada com métodos e técnicas, e ainda, utilizando uma empolgante trilha sonora, alegria, motivação e com os diferentes objetivos, vem sendo uma experiência marcante em todos os recantos (Albuquerque, 2006).

Em relação à experiência acima relatada, foi aplicado um formulário da plataforma Google Forms contendo perguntas abertas e fechadas, onde na pesquisa participaram 30 indivíduos do sexo feminino em sua totalidade, com idade média variando entre 30 e 50 anos de idade, e todos com experiência na prática do Spinning, onde os indivíduos foram questionados sobre a experiência de realizar treinos de Spinning remotos em casa durante o período de isolamento social. A pesquisa seguiu os preceitos éticos descritos na Resolução no 510 do Conselho Nacional de Saúde (CNS), promulgada em 2016. Ela determina procedimentos éticos específicos para investigações com seres humanos, que utilizam abordagens das Ciências Humanas e Sociais (CHS). Destaca ainda em seu Artigo $1^{\circ}$, Parágrafo Único que a referida resolução indica que pesquisas de opinião pública com participantes não identificados não necessitam ser enviadas ao Comitê de Ética em Pesquisa com Seres Humanos.

\section{Resultados e Discussão}

Após a aplicação dos questionários foram encontrados os seguintes resultados: Quando questionados sobre a experiência de realizar treinos remotos de Spinning, 68,2\% consideraram a experiência EXCELENTE, 9,1\% julgaram a experiência ÓTIMA e 22,7\% como BOA. Segundo Pitanga et. al. (2020) no caso de academias e/ou ginásios estarem fechados para os usuários, a atividade física deverá ser mantida, quando possível, em ambientes abertos. Neste caso as pessoas devem priorizar as atividades feitas individualmente, sempre evitando aglomerações ou até mesmo pequenos grupos. Se todas as possibilidades citadas anteriormente estiverem com restrições a atividade física deve ter continuidade em casa, preferencialmente com auxílio de procedimentos tecnológicos, tais como vídeos com séries de exercícios, aplicativos e orientação profissional on-line. Ferreira et. al. (2020) corroboram, e afirmam que para melhor enfrentamento deste momento de isolamento social, propõe à população alguns comportamentos e atitudes que ajudarão na manutenção da vida ativa e melhora da saúde física e mental: realizar atividades físicas prazerosas, explorando da melhor forma os espaços e materiais disponíveis. 
Ainda em relação a pesquisa, a maioria dos participantes realizavam treinos remotos de Spinning 2 ou 3 vezes por semana, representando $18,2 \%$ e $50 \%$ respectivamente, $4,5 \%$ relataram se exercitar 7 dias por semana, $9,1 \%$ se exercitam 5 dias por semana, 18,2\% se exercitaram 1 vez por semana. Segundo Pitanga et. al. (2019) a duração e intensidade da atividade física necessária para que os benefícios possam ocorrer, recente publicação sugeriu que atividades na intensidade modera/vigorosa com duração de 180 a 300 minutos por semana para homens e na intensidade moderada/vigorosa com duração de 150 a 300 minutos por semana para mulheres seriam as mais adequadas para promover os benefícios para a saúde cardiovascular e metabólica. Hammami et al. (2020) apresentam informações uteis para a realização diária de atividade física em casa para adultos e idosos (> 17 anos), recomenda-se atividade física diária por $75 \mathrm{~min} / \mathrm{semana}$ com exercícios aeróbicos de intensidade vigorosa ou $150 \mathrm{~min} / \mathrm{semana}$ de intensidade aeróbica moderada, com fortalecimento muscular e ósseo duas vezes por semana. Recomendam ainda que as pessoas permaneçam ativas exercitando-se em casa. Neste sentido, poderão ser aplicadas diferentes tipos de atividades, incluindo exercícios aeróbicos usando bicicletas estacionárias ou ergômetros de remo, treinamento de força com peso corporal e exercícios baseados em dança.

Quanto ao uso da plataforma digital (lives no Instagram) para acompanharem as aulas, a maioria afirmou ser excelente ou ótima (18,2\% e 50\% respectivamente), 27,3\% consideraram uma BOA ferramenta e 4,8\% consideram a ferramenta REGULAR. Segundo Pintanga et. al. (2020) caso de a atividade física ter que ser realizada em casa sugere-se exercícios de fortalecimento muscular (agachamentos, flexões, abdominais, entre outros), alongamentos, exercícios de equilíbrio e subida/descida de escadas, de preferência com auxílio de procedimentos tecnológicos, tais como vídeos com séries de exercícios, aplicativos e orientação profissional on-line.

Outro dado importante é que, segundo a mesma pesquisa, todos os usuários recomendariam a outras pessoas participarem de treinos remotos de Spinning em casa; cabe destacar também que, dentre as diversos benefícios percebidos pelos participantes da pesquisa, o mais relatado foi a comodidade de realizar as sessões de exercícios físicos em casa a qualquer dia e momento, além da segurança de estarem em suas próprias casas; e por fim vivenciarem o lazer, combate ao sedentarismo, manutenção e/ou melhoria da composição corporal, adoção de hábitos saudáveis relacionados a um estilo de vida ativo, melhoria do estado geral de saúde, melhoria do humor, equilíbrio emocional e afetivo. Sabe-se que a prática regular de exercícios físicos em estado de isolamento social pode trazer ao organismo diversos benefícios fisiológicos, além de fortalecer a imunidade no combate ao COVID-19 (Krinski, 2010), corroboram com a discussão Lin et. al. (2016) e Pitanga et. al. (2019) Os benefícios da atividade física regular sobre a saúde cardiovascular/metabólica são amplamente divulgados na literatura há bastante tempo. A atividade física apresenta associação inversa com níveis pressóricos, diabetes, alterações lipídicas e risco de doença arterial coronariana e outros eventos cardiovasculares.

\section{Conclusão}

Pode-se julgar que a experiência acima citada foi muito benéfica e saudável para os participantes, e ainda, que devem ser encorajadas ações que estimulem a prática de exercícios físicos em casa nesse período de pandemia, nas diversas faixas etárias, sempre na tentativa de manter as pessoas ativas e assim, proporcionar uma melhor condição de saúde física e emocional, assim como, a redução do risco do agravamento ou aparecimento de doenças. Também importante ressaltar que a prática regular de exercícios físicos (em casa ou em espaços públicos, com respeito ao distanciamento e uso de constante de máscara), uma alimentação adequada, a qualidade de sono e o equilíbrio emocional são fatores indispensáveis para o auto cuidado em tempos de pandemia e isolamento social.

\section{Referências}

Albuquerque, F. (2006). Treinamento em bicicletas estacionárias. PH Editora.

American College of Sports Medicine. (2020). Staying Active During the Coronavirus Pandemic. https://www.exerciseismedicine.org/assets/page_d ocument s/EIM_Rx\%20for\%20Health_\%20Staying\%20Active\%20During\%20Coronavirus\%20Pandemic.pdf. 
Brasil, MS. Cartilha Coronavírus. https://www.saude.gov.br/images/pdf/2020/April/07/Cartilha- Coronavirus-Informacoes-.pdf.

Brasil, MS. (2015). Saúde Brasil 2014: uma análise da situação de saúde e das causas externas/Ministério da Saúde, Secretaria de Vigilância em Saúde, Departamento de Vigilância de Doenças e Agravos Não Transmissíveis e Promoção da Saúde. Brasília.

Ferreira, M. J., Irigoyen, M. C., Consolim-Colombo, F., Saraiva, J. F. K., \& De Angelis, K. (2020). Vida Fisicamente Ativa como Medida de Enfrentamento ao COVID-19. Arq Bras Cardiol http://publicacoes.cardiol.br/portal/abc/portugues/aop/2020/AOP_2020-0235.pdf

Hammami, A., Harrabi, B., Mohr, M., \& Krustrup, P. (2020). Physical activity and coronavirus disease 2019 (COVID-19): specific recommendations for home-based physical training. Manag Sport Leis 20;0(0):1-6. https://doi.org/10.1080/23750472.2020.1757494

Krinski, K. et al. (2010). Efeitos do exercício físico no sistema imunológico. RBM; 67, 7.

Leandro, C. G. et al. (2007). Mecanismos adaptativos do sistema imunológico em resposta ao treinamento físico. Revista Brasileira de Medicina do Esporte; 13, 343-348.

Lin, X., Alvim, S. M., Simoes, E. J., Bensenor, I., Barreto, S., Schimidt, M., et al. (2016). Leisure time physical activity and cardio-metabolic health: results from the Brazilian Longitudinal Study of Adult Health (ELSA-Brasil). J Am Heart Assoc.; 5(6):003337.

Ornell, F., Schuch, J. B., Sordi, A. O., \& Kessler, F. H. P. (2020). Pandemia de medo e COVID-19: impacto na saúde mental e possíveis estratégicas. Revista Debates in Psychiatry.

Pires, R. R. C. (2020). Os efeitos sobre grupos sociais e territórios vulnerabilizados das medidas de enfrentamento à crise sanitária da covid-19: propostas para o aperfeiçoamento da ação pública: Nota Técnica. IPEA. Brasília.

Pintanga, F. J. G., Beck, C. C., \& Pintanga, C. P. S. (2020). Atividade Física e Redução do Comportamento Sedentário durante a Pandemia do Coronavírus. Arq Bras Cardiol. 114(6):1058-1060

Pitanga, F. J. G., Pitanga, C. P. S., \& Beck, C. C. (2019). Physical Activity for the Prevention of Cardiometabolic Diseases: how much is Required? Curr Res Diabetes \& Obes J. 9(4):

Pitanga, F. J. G., Matos, S. M. A., Almeida, M. D. C. C., Patrão, A. L., Molina, M. D. C. B., \& Aquino, E. M. (2019). Association between leisure-time physical activity and sedentary behavior with cardiometabolic health in the ELSA-Brasil participants. SAGE Open Med.7:1-9.

Rosa, C., Bicudo, L. F. P., \& Vaisberg, M. W. (2020). Influências do exercício na resposta imune. Revista Brasileira de Medicina do Esporte; 8, 167-172.

Santos, V. C., \& Santos, A. C. (2010). Exercício Físico e seus Efeitos Sobre o Sistema Imune dos Idosos. Saúde e Pesquisa; 3.

Sociedade Brasileira de Medicina do Exercício e do Esporte. (2020). Informe da Sociedade Brasileira de Medicina do Exercício e do Esporte sobre exercício físico e o coronavírus (COVID-19). http://www.medicinadoesporte.org.br/informes-da-sbmee-sobre-coronavirus-e-exercicio-fisico/.

Terra, R. et al. (2012). Efeito do exercício no sistema imune: resposta, adaptação e sinalização celular. Revista Brasileira de Medicina do Esporte; 18, 208214.

The Guardian. (2020). How physical exercise makes your brain work better. https://www.theguardian.com/education/2016/jun/18/how-physical-exercisemakes-your-brain-work-better.

Werneck, G. L., \& Carvalho, M. S. (2020). A pandemia de COVID-19 no Brasil: crônica de uma crise sanitária anunciada. Caderno de Saúde Pública. 36.

World Health Organization. (2020). Guia para atividade física na quarentena: seja ativo e saudável nesta quarentena.

World Health Organization. (2020). Coronavirus disease 2019 (COVID-19): situation report, 72. 